Erratum

\title{
Prediction of serum cortisol response to dexamethasone in normal volunteers: A multivariate approach
}

Roland J. Branconnier, Gregory F. Oxenkrug, Iain McIntyre, Nunzio Pomara, Nancy E. Harto, and Samuel Gershon

Psychopharmacology (1984) 84: 274-275

One of the co-authors of the above article was inadvertently omitted. The authors should have been listed as follows:

Roland J, Branconnier, Gregory F. Oxenkrug, Iain McIntyre, Nunzio Pomara, Nancy E. Harto, Samuel Gershon, and Michael Stanley 